\title{
REMARK ON OZEKI INEQUALITY FOR CONVEX POLYGONS
}

\section{Igor Ž. Milovanović, Emina I. Milovanović and Tatjana Z. Mirković}

Abstract. This paper gives proof of a discrete inequality that represents Ozeki's inequality for convex polygons and its converse. The proof is based on determining eigenvalues of one nearly tridiagonal symmetric matrix.

Mathematics subject classification (2010): 26D15, 26D07.

Keywords and phrases: Convex polygons, Opial inequality, Ozeky inequality.

\section{REFERENCES}

[1] R. P. Agarwal, Difference equations and inequalities: Theory, Methods and Applications, Mercel Denker, New York-Basel-Hong Kong, 2000.

[2] S. S. CHENG, T. T. LU, Qualitative properties of matrices appearing in several mathematical models, Proc. Math. Sci. Couna. ROC (A), Vol. 25, 5 (2001), 269-283.

[3] M. DincA, Proof Wolstenholme-Lenhard ciclic inequality for real numbers and L. Fejes Tóth conjecture, VIXra:1008.0030, 2010 (http://vixra.org/gom/)

[4] P. ERdös, Problem 3740, Amer. Math. Monthly, 42 (1935), 396.

[5] H. C. LENHARD, Verallgemeinerung und Vershärfung der Erdös-Mordellschen Ungleichung für polygone, Arch. Math., 12 (1961), 311-314.

[6] L. LosonCZI, Eigenvalues and eigenvectors of some tridiagonal matrices, Acta. Math. Hung., Vol. 60, 3 (1992), 309-322.

[7] G. V. Milovanovic, I. Ž. Milovanović, Some discrete inequality of Opial's type, Acta. Sci. Math. (Szeged), 47 (1984), 413-417.

[8] D. S. Mitrinović, J. E. PeČArić, V. VOlEneC, Recent advances in geometric inequalities, Kluwer Academic Publishers, Dordrecht, 1989.

[9] L. J. Mordell, D. F. Barrow, Solution of problem 370, Amer. Math. Monthly, 44 (1937), 252 254.

[10] N. Ozeki, On P. Erdös inequality for the triangle, J. College Arts Sci., Chiba Univ., 2 (1957), $247-$ 250.

[11] S. Wu, L. Debnath, Generalization of the Wolstenholme cyclic inequality and its application, Comput. Math. Appl., 53, 1 (2007), 104-114.

[12] J. Wolstenholme, A book of matheamtical problems, Cambridge, London, 1867.

[13] A. Vandanjav, B. TserendorJ, B. UndrakH, On some weighted Erdös-Mordell's type inequalities for polygons, Int. J. Geometry, Vol. 1, 2 (2012), 15-21. 